\title{
ANÁLISIS DE CUANTIFICACIÓN DE PLOMO EN SUELOS DE PARQUES RECREACIONALES DE LA CIUDAD DE LIMA - PERÚ
}

\section{ANALYSIS OF LEAD QUANTIFICATION IN RECREATIONAL PARK SOILS OF THE CITY OF LIMA - PERU}

\author{
Lily Tello ${ }^{1}$, Jorge Jave ${ }^{2}$ y Juan Guerrero ${ }^{3}$
}

\begin{abstract}
Resumen
Los Grandes Parques de Lima Metropolitana (GPLM) son áreas verdes donde habitan diversidad de especies de origen vegetal y animal. Los GPLM, áreas recreacionales localizadas en la cuenca del río Rímac considerados en este estudio fueron los siguientes: Bosque El Olivar $\left(12^{\circ} 06^{\prime} 04^{\prime \prime} \mathrm{S}, 7^{\circ} 02^{\prime} 05^{\prime \prime} \mathrm{O}\right)$, Campo de Marte $\left(12^{\circ} 04^{\prime} 06^{\prime \prime} \mathrm{S}, 77^{\circ} 02^{\prime} 29^{\prime \prime} \mathrm{O}\right)$, Pentagonito $\left(12^{\circ} 06^{\prime} 01^{\prime \prime} \mathrm{S}, 76^{\circ} 59^{\prime} 35^{\prime \prime} \mathrm{O}\right)$, Zoológico de Huachipa $\left(12^{\circ} 00^{\prime} 52^{\prime \prime} \mathrm{S}, 76^{\circ} 53^{\prime} 52^{\prime \prime} \mathrm{O}\right)$, Las Leyendas $\left(12^{\circ} 07^{\prime} 14^{\prime \prime} \mathrm{S}, 77^{\circ} 05^{\prime} 12^{\prime \prime} \mathrm{O}\right)$ y Kennedy $\left(12^{\circ} 07^{\prime} 18^{\prime \prime} \mathrm{S}, 77^{\circ} 01^{\prime} 49^{\prime \prime} \mathrm{O}\right)$. La presencia del plomo en los suelos representa un peligro potencial para la salud de los habitantes y usuarios, por lo que el objetivo de esta investigación fue cuantificar el contenido total de plomo en los suelos de los GPLM. Para ello, se realizaron muestreos de identificación, análisis físicos (textura) y químicos ( $\mathrm{pH}$, conductividad eléctrica, materia orgánica, capacidad de intercambio) en muestras de suelos de cada parque. En aquellos parques que presentaron contaminación por plomo se realizó un muestreo de detalle con análisis adicionales de plomo total. Los valores promedio de plomo total en los suelos de los GPLM Bosque El Olivar (170 ppm Pb), Campo de Marte (226 ppm Pb) y Pentagonito (159 ppm $\mathrm{Pb}$ ) se encontraron por encima de lo permitido por los Estándares de Calidad Ambiental (ECAPerú:140 ppm). En el parque Las Leyendas se determinó que ocho de las 98 hectáreas estaban contaminadas (234.5 ppm Pb) y en el Zoológico de Huachipa 0.7 de las 11 hectáreas (266 ppm Pb) sobrepasaron el limite ECA; mientras que el parque Kennedy no sobrepasó los valores de ECA (5678 ppm $\mathrm{Pb}$ ).
\end{abstract}

Palabras clave: metales pesados, suelos contaminados, áreas verdes urbanas.

\begin{abstract}
The recreational parks of Lima city (GPLM) are green areas where diverse species of animals and plants live. These are also recreational areas located in the Rímac river basin. The GPLM considered were Bosque El Olivar $\left(12^{\circ} 06^{\prime} 04^{\prime \prime} \mathrm{S}, 77^{\circ} 02^{\prime} 05^{\prime \prime} \mathrm{O}\right)$, Campo de Marte $\left(12^{\circ} 04^{\prime} 06^{\prime \prime} \mathrm{S}\right.$,

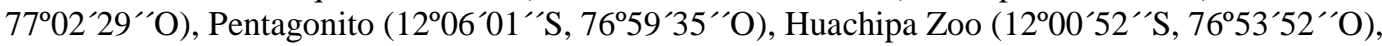
Las Leyendas $\left(12^{\circ} 07^{\prime} 14^{\prime \prime} \mathrm{S}, 77^{\circ} 05^{\prime} 12^{\prime \prime} \mathrm{O}\right)$ and Kennedy $\left(12^{\circ} 07^{\prime} 18^{\prime \prime} \mathrm{S}, 77^{\circ} 01^{\prime \prime} 49^{\prime \prime} \mathrm{O}\right)$. Lead presence in the soils constitutes a potential danger to the health of the inhabitants and users. The objective of this study was to quantify the total lead content in the GPLM soils. Samplings were performed for identifications, physical (texture) and chemical ( $\mathrm{pH}$, electric conductivity, organic matter, CIC) analysis in each park. Those parks that showed lead contamination were subjected to detailed samplings with additional total lead analysis. The mean values of total lead content in soils of the GPLM Bosque Olivar (170 ppm Pb), Campo de Marte (226 ppm Pb) and Pentagonito (159 ppm Pb) were found to be above those allowed by the Environmental Quality Standards (ECA-Peru: 140 $\mathrm{ppm})$. At Las Leyendas park eight of the 98 hectares were contaminated $(234.5 \mathrm{ppm} \mathrm{Pb})$ and at Huachipa Zoo 0.7 of 11 hectares were contaminated too (266 ppm Pb), while Kennedy Park did not exceed the ECA (56 ppm Pb).
\end{abstract}

Key words: lead, contaminated soils, urban green areas.

\section{Introducción.}

En Lima Metropolitana no se tienen registros de niveles de plomo en los suelos de los grandes parques. Se sabe que el Estándar de Calidad Ambiental ([ECA] para suelos de Perú (DS 011 - 2017-Ministerio del Ambiente [MINAM]) recomienda que el nivel de plomo en los suelos urbanos se encuentre por debajo de 140 ppm en parques.

Los grandes parques de Lima Metropolitana (GPLM) son frecuentados por personas de diversas edades y para múltiples fines. El Campo de Marte, ubicado en el distrito de Jesús María, con $375.2 \mathrm{~km}^{2}$ de área, es muy conocido por realizarse desfiles militares 
y escolares, ferias artesanales y/o de alimentos típicos y posee ocho monumentos históricos. El Bosque El Olivar, ubicado en el distrito de San Isidro, con $230 \mathrm{~km}^{2}$ de área, fue declarado monumento nacional el 16 de diciembre del 1959, con 1600 olivos dentro de un total de 1946 árboles y con ejemplares de 350 años de antigüedad y 35 especies de aves estacionales más 22 nativas; por lo cual, en el año 2017 fue declarado Área de Conservación Ambiental. El parque El Pentagonito, ubicado en el distrito de San Borja, con un área aproximada de $5 \mathrm{~km}^{2}$, básicamente es un área verde que rodea el Cuartel General del Ejército del Perú y donde los vecinos trotan y realizan ejercicios. El parque Kennedy, ubicado en el distrito de Miraflores, con un área de $25 \mathrm{~km}^{2}$, es un parque donde constantemente se realizan cambios estructurales con movimientos y cambios de tierras debido a que su característica es ser lugar de actividades culturales y comerciales. Los suelos de Campo de Marte y Bosque El Olivar tienen cientos de años y el Pentagonito alrededor de 45 años. Estos parques colindan con vías de comunicación de acceso público y/o privado, incluso en el caso de Bosque El Olivar es atravesado por pistas automotrices con tráfico vehicular la mayor parte del tiempo (7.00 am a $9.00 \mathrm{pm}$ ) y hay casas de la época colonial. Se tiene como referencia que el uso de gasolina y pintura con plomo en su composición fue una fuente importante de este metal pesado en los suelos urbanos (Witzling et al., 2011), por lo que se prohibió su uso como aditivo. Esto se cumplió en el país a partir del 2005 (Arosquipa \& Villegas, 2013; OSINERGMIN, 1998; OSINERGMIN, 2012).

Castillo (2010) encontró en las bermas de suelos urbanos de Lima Metropolitana, ubicados en lugares con mayor afluencia vehicular, una mayor concentración de plomo en los primeros centímetros de suelos con niveles promedio de $189 \mathrm{ppm} \mathrm{Pb}$ y con valores picos de $412 \mathrm{ppm}$ de $\mathrm{Pb}$ en los cruces de avenida Aviación con avenida San Borja. Por ser el plomo un elemento pesado de lenta movilidad, se queda en la superficie del suelo por muchos años.

El parque Zoológico de Huachipa, ubicado en la margen izquierda del río Rímac en el distrito de Ate Vitarte, con un área de $110 \mathrm{~km}^{2}$, posee más de 300 especies, entre autóctonas y foráneas, dando un total de alrededor 1000 especímenes, la mayoría nativos como pingüinos de Humbolt, pavas aliblancas, lobos de mar, entre otros. Por este motivo, la Cadena Internacional Telemundo lo ha considerado dentro de los 11 mejores parques de América Latina (El Comercio, 2015). En los meses de diciembre a marzo, por tratarse de época de lluvias en la sierra, el río normalmente se desborda inundando la zona del Bosque de Aves y la entrada principal del parque. Esta zona se caracteriza por actividades de desarrollo industrial con fábricas de ropa y pinturas lo cual podría liberar metales pesados, que ocasiona contaminación en los suelos (Ji et al., 2011; Pottier et al., 2015). La Autoridad Nacional del Agua
(2012) realizó un monitoreo del agua de la cuenca del río Rímac encontrando en su parte baja (Ate Vitarte) los mayores problemas de contaminación por plomo, probablemente por los sedimentos transportados, debido a que el plomo no es soluble en el agua sino que se encuentra adsorbido a las partículas (Xu et al., 2017).

El parque de Las Leyendas ubicado en el distrito de San Miguel, con un área de $980 \mathrm{~km}^{2}$ fue inaugurado en 1964 y posee 118 especies de mamíferos, 33 de aves y 4 reptiles en costa, 118 especies de mamíferos y 93 de aves en Sierra, 18 especies de Selva y 19 especies en zona internacional. La zona denominada "Pampa Galeras" tiene una extensión de ocho hectáreas, es una depresión que por los años de 1730 era un estanque de agua de la Hacienda Maranga (Narváez, 2013). En ambos se promueven acciones para la conservación de la naturaleza y biodiversidad, son hábitat de una gran diversidad de especies y reciben miles de visitantes por mes.

Temperaturas mayores a $20^{\circ} \mathrm{C}$, humedad por debajo de la capacidad de campo del suelo, incrementan la suspensión y movimiento de pequeñas partículas de suelo por acción del viento y que pueden demorar de 7 a 14 días en depositarse, y coinciden con los mayores niveles de plomo en la sangre humana, 3 a $7 \mu \mathrm{g} / \mathrm{dl}$ por cada 1000 ppm de incremento en la concentración de plomo en el suelo (Doyle, 1998; Witzling et al., 2011).

El plomo es un elemento perjudicial para la salud humana, especialmente para mujeres gestantes y niños pequeños (Yang-Guang \& Yan-Peng, 2016), quienes son la población urbana más sensible y vulnerable a ambientes contaminados (Tepanosyan et al., 2017). En el caso de animales, este elemento ocupa el lugar del calcio y zinc, dañando células y afectando el sistema cardiovascular, generando complicaciones gastrointestinales, anemia y afectando funciones del sistema nervioso central (Sanín et al., 1998). Bovinos de año y medio de edad que fallecieron con síntomas de problemas neurológicos presentaron $17.6 \mathrm{ppm}$ de plomo en sus vísceras y $0.51 \mathrm{ppm}$ de plomo en su sangre (Martínez \& Villafañe, 1999). En las plantas puede ocasionar fitotoxicidad porque no se realizan funciones fisiológicas y bioquímicas (Amari, 2017).

Por lo expuesto, el objetivo del presente estudio fue cuantificar el contenido total de plomo en los suelos de los GPLM.

\section{Materiales y métodos.}

Muestreos de Identificación y de Detalle.

La elección de técnicas y lugares de muestreo, manejo de muestras y medidas de seguridad ocupacional, siguieron las pautas dadas en la Guía para Muestreo de Suelos (MINAM, 2014), en el marco de los Estándares de Calidad Ambiental (ECA) para suelo.

La investigación se realizó durante el 2016 en seis de los GPLM (Figura 1). Al no tener conocimiento previo de la distribución del contaminante plomo en el suelo, el número mínimo de puntos de muestreo estuvo 


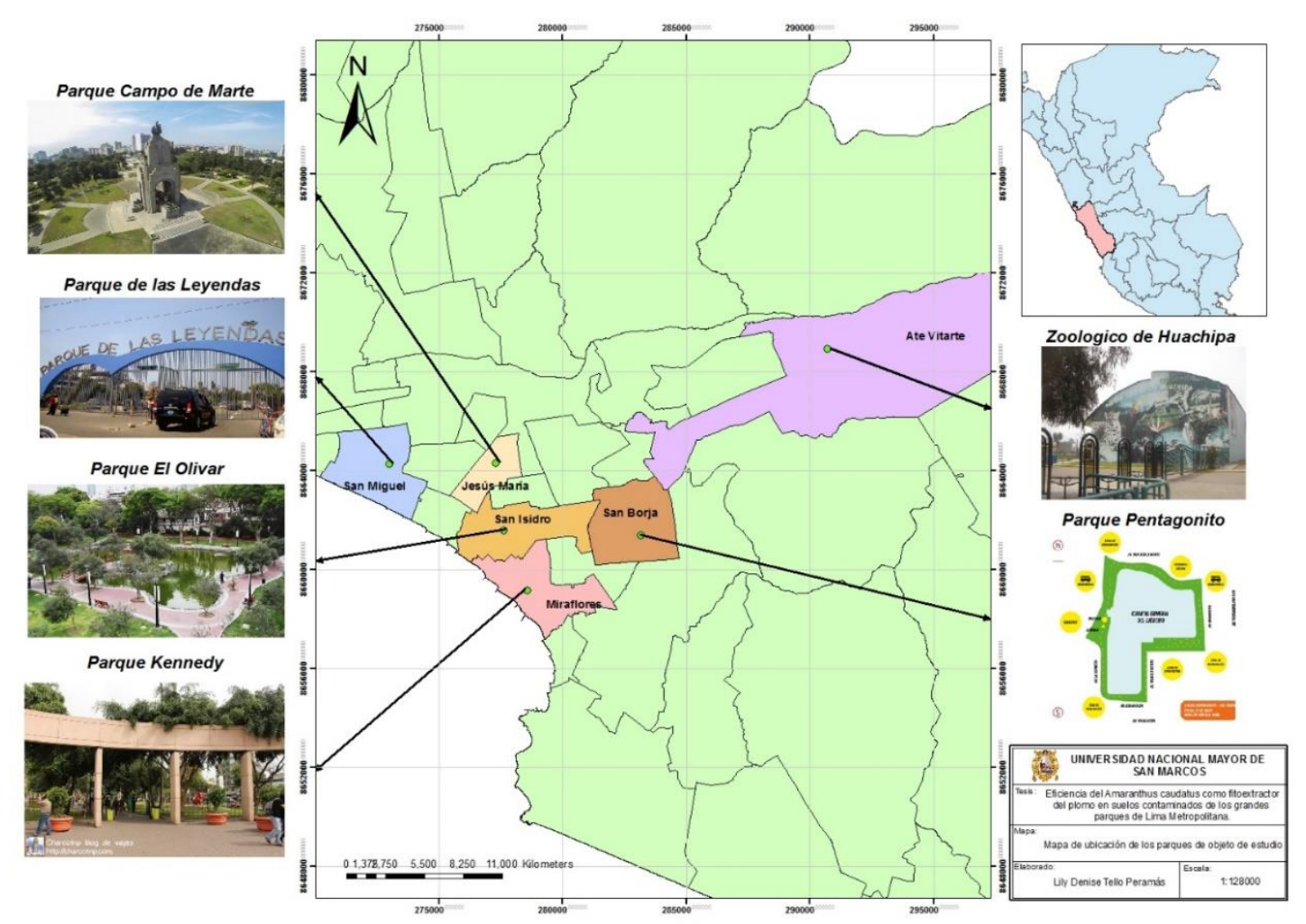

Figura 1. Mapa del Departamento de Lima con la ubicación de los seis parques.

en función del área de cada parque siendo éste el Muestreo de Identificación (MI) (Tabla 1). El objetivo fue obtener muestras representativas con el fin de determinar si el suelo supera o no los ECA para suelos de parques (MINAM, 2017). Se realizó la georreferenciación en gabinete en el programa Google Earth, utilizando el sistema de coordenadas Universal Transversal Mercator (UTM) zona 18 Sur, datum World Geodetic system 1984 (WGS 84), pasando las coordenadas al GPS y siguiendo el patrón de muestreo de rejillas regulares. En campo, se realizaron correcciones, dependiendo de la accesibilidad y homogeneidad del lugar elegido. Se colectaron muestras de suelo de $150 \mathrm{~g}$ a $10 \mathrm{~cm}$ de profundidad por ser la capa de contacto oral o dermal del contaminante y se muestrearon lo más equidistantes entre ellos de acuerdo a lo georreferenciado.

El número de muestras contaminadas del muestreo de identificación (Anexo 1) determinó el número de muestreos adicionales a realizar para el muestreo de detalle (MD) (Tabla 1); teniendo en cuenta que el número mínimo de puntos de muestreo representan el "mínimo de calidad" y por ello deberán tomarse muestras de un número igual o mayor de puntos de muestreo (MINAM, 2014).

En el laboratorio se empleó el método de digestión ácida, para cada muestra de suelo. Se pesaron $5 \mathrm{~g}$ en un matraz Erlenmeyer, se adicionaron $20 \mathrm{ml}$ de la mezcla ácida (solución de ácido nítrico y ácido perclórico en proporción 5:1). El matraz se colocó en la plancha de digestión por aproximadamente $2 \mathrm{~h}$ a una temperatura de $180-200^{\circ} \mathrm{C}$. Se dejó enfriar para luego transferir la muestra a una fiola y enrasar con agua desionizada a 25 ml. Se trasvasó a un tubo de ensayo y se agitó. Se tomó una alícuota de $1 \mathrm{ml} \mathrm{y} \mathrm{se} \mathrm{adicionaron} 9 \mathrm{ml}$ de agua desionizada. Se leyó la concentración de plomo en un espectrofotómetro de absorción atómica marca Perkin Elmer, modelo AAnalyst 200, Estados Unidos, 2005. Para el control de calidad se realizaron réplicas de muestras al azar, el estándar de referencia que se utilizó fue agua pura y el límite de detección $0.1 \mathrm{mg} \mathrm{kg}^{-1}$. Determinación de las Características de los Suelos.

En cada parque se realizaron muestreos simples en profundidad de $0-10 \mathrm{~cm}$ de $20 \mathrm{~g}$ cada una formando una

Tabla 1. Número de muestras para el MI y MD en función al área.

\begin{tabular}{|c|c|c|c|c|}
\hline Parque & $\begin{array}{l}\text { Área } \\
\text { (ha) }\end{array}$ & $\begin{array}{l}\text { Número } \\
\text { de } \\
\text { muestras } \\
\text { en MI } \\
\text { (depende } \\
\text { del área) }\end{array}$ & $\begin{array}{c}\text { Muestras } \\
\text { mayor a } \\
140 \mathrm{ppm} \\
\text { de } \mathrm{Pb}\end{array}$ & $\begin{array}{l}\text { Número } \\
\text { de } \\
\text { muestras } \\
\text { en MD }\end{array}$ \\
\hline $\begin{array}{l}\text { Campo de } \\
\text { Marte }\end{array}$ & 37.52 & 42 & 34 & 65 \\
\hline $\begin{array}{l}\text { Bosque El } \\
\text { Olivar }\end{array}$ & 23.00 & 38 & 17 & 34 \\
\hline Pentagonito & 0.50 & 6 & 1 & 6 \\
\hline Kennedy & 2.50 & 19 & 1 & 13 \\
\hline $\begin{array}{l}\text { Las } \\
\text { Leyendas }\end{array}$ & 98.00 & 50 & 2 & 6 \\
\hline Huachipa & 11.00 & 33 & 10 & 44 \\
\hline
\end{tabular}
(2014). 
muestra compuesta. El patrón de muestreo fue rejillas regulares. Las seis muestras compuestas se secaron al aire y se pasaron por un tamiz de malla de $2 \mathrm{~mm}$. Se determinaron: Textura con el método del hidrómetro y las lecturas de las partículas en suspensión se realizaron con el hidrómetro de Bouyoucos. Conductividad eléctrica (CE) en extracto de saturación, a $100 \mathrm{~g}$ de suelo se le añadieron $20 \mathrm{ml}$ de agua desionizada, se removió y se agregó más agua a cada muestra hasta llegar a saturación. Finalmente con un sistema de vacío se realizó la extracción del líquido y la CE se midió con un conductímetro. También se midió directamente en el suelo luego de 30 minutos del riego. Ambas medidas se realizaron con el equipo Electrical Equipment for Measurement, Control, and Laboratory Use de Fieldscout Item 2265FS y solución estándar 2764 uS/cm. Capacidad de intercambio catiónico (CIC), mediante el extracto con acetato de amonio $1 \mathrm{~N} \mathrm{pH} 7$ (Jackson, 1964). Para el pH se hizo una mezcla sueloagua (relación 1:1) que se agitó y dejó en reposo por 10 minutos, la medición se realizó con el pH-metro Soil Stik de Fieldscout 2105. La materia orgánica fue determinada por el método de Walkley and Black (1934).

Análisis estadístico.

Se utilizaron dos pruebas estadísticas; la Prueba T para una muestra, para los parques con un número de muestreos menor a 40, donde si $\mathrm{T}_{\text {práctico }}<\mathrm{T}_{\text {crítico }}$ no existen diferencias significativas y la Prueba $\mathrm{Z}$ para los parques cuyo número de muestreos fue mayor a 40 donde los valores de $\mathrm{Z}_{0.025}$ fueron -1.96 y 1.96 y se comparó $\mathrm{p}$ con $\alpha(0.05)$, donde si $\mathrm{p}<\alpha$ existen diferencias significativas. El valor de prueba fue 140 ppm de plomo total que corresponde al límite máximo permisible por los ECA de suelos 2017.

En el caso de los parques Zoológico de Huachipa (PZH) y Las Leyendas (PLL) los valores de plomo total en suelos se dividieron de dos grupos focalizados por espacios continuos, con eventos peculiares como ex laguna en el PLL y zona inundable en el PZH y por las marcadas diferencias respecto al límite máximo permisible de $140 \mathrm{ppm}$.

\section{Resultados y discusión.}

Los resultados de plomo total en cada punto de muestreo de los diferentes parques se registran en el Anexo 1. En el caso particular de los PLL y PZH se encontró que los valores que sobrepasan el máximo permitido (140 ppm) se encontraban focalizados. En Las Leyendas, el área contaminada fue la zona de "Pampa Galeras" (media de 234.47 ppm de Pb total) en un espacio de ocho hectáreas, corresponde a una depresión que por los años de 1730 era un estanque de agua de la Hacienda Maranga y que fue el último lugar donde desembocaba el agua del canal Huatica (Narváez, 2014), siendo hasta el año 2009 un lecho de laguna antigua y luego al desaparecer el agua quedaron los sedimentos depositados desde la época colonial y

Tabla 2. Prueba "T" para una muestra con valor de prueba $=140 \mathrm{ppm} \mathrm{Pb}$ de las concentraciones totales de plomo en los suelos de los GPLM.

\begin{tabular}{lrcccc}
\hline & $\begin{array}{c}\text { Media } \\
\mathrm{ppm} \mathrm{Pb}\end{array}$ & $\mathrm{T}_{\text {práctico }}$ & Tcrítico & $\mathrm{n}$ & $\begin{array}{c}\text { Sig } \\
\text { (bilateral) }\end{array}$ \\
\cline { 2 - 6 } Pentagonito & 159.15 & 4.68 & 2.2 & 12 & 0.0001 \\
Kennedy & 55.52 & -25.89 & 2.04 & 32 & 0.000 \\
$\begin{array}{l}\text { Pampa } \\
\text { Galera-PLL }\end{array}$ & 234.47 & 3.433 & 2.45 & 7 & 0.014 \\
$\begin{array}{l}\text { Bosque de } \\
\text { Aves-PZH }\end{array}$ & 266.41 & 5.436 & 2.14 & 15 & 0.000 \\
\hline $\mathrm{n}$. Tamaño & muestral; & &
\end{tabular}

n: Tamaño muestral; $\mathrm{T}_{\text {práctico: }}$ encontrado con los datos experimentales; $\mathrm{T}_{\text {crítico: }}$ obtenido en tabla.

que podría explicar el alto valor de plomo total presente en los suelos. La prueba $\mathrm{T}$ indica diferencias significativas donde $\mathrm{T}_{\text {práctica }}>\mathrm{T}_{\text {crítica }}($ Tabla 2). En el Zoológico de Huachipa, la zona de bosque de aves tiene una extensión aproximada de 0.7 ha, y la prueba $\mathrm{T}$ indica diferencias significativas donde $\mathrm{T}_{\text {práctica }}>\mathrm{T}_{\text {crítica }}$ (media de $266.41 \mathrm{ppm}$ de $\mathrm{Pb}$ total), es decir, se tiene contaminación con plomo lo cual podría ser por los sedimentos dejados por el agua del río Rímac en época de avenida cuando se desborda e inunda la zona de bosque de aves depositando sedimentos; además, se encontró que los suelos que colindan con el surco por donde pasa el agua del río Rímac bajo la jurisdicción de la junta de regantes, presentan contaminación, mientras que el resto del parque que se riega con sistema de goteo/aspersión no tiene contaminación probablemente porque los sedimentos no pasan por los sistemas de riego. En ambos parques la contaminación se explica porque el plomo no es soluble en agua; por tanto, son los sedimentos transportados por el agua, y que se quedan en los suelos de los parques, los que podrían estar causando la contaminación, concordando con los resultados obtenidos por Moronta-Riera \& RiverónZaldivar (2016). En el resto de área de los parques Huachipa y Leyendas donde $\mathrm{p}<\alpha$ y $\mathrm{Z}_{\text {crítico }}$ es negativo, lo que indica que no hay contaminación de plomo en los suelos (Tabla 3). Los suelos son irrigados con agua de pozo. En el parque Kennedy $\left(\mathrm{T}_{\text {práctica }}<\mathrm{T}_{\text {crítico }}\right)$, el nivel de plomo en los suelos también está por debajo de los límites permisibles de 140 ppm (MINAM, 2017).

La prueba $\mathrm{Z}$ en las muestras de suelos para los parques Campo de Marte y Bosque El Olivar así como prueba $\mathrm{T}$ para el parque Pentagonito, resultaron con diferencias significativas, es decir, se encuentran

Tabla 3. Prueba " $Z$ " para un valor crítico entre -1.96 y 1.96 , con valor de prueba $=140 \mathrm{ppm} \mathrm{Pb}$ de las concentraciones totales de plomo en los suelos de los GPLM.

\begin{tabular}{|c|c|c|c|c|}
\hline & $\begin{array}{c}\text { Media } \\
\text { ppm } \mathrm{Pb}\end{array}$ & $Z_{\text {práctico }}$ & $\mathrm{n}$ & $\mathrm{p}$ (bilateral) \\
\hline Campo de Marte & 225.59 & 10.21 & 107 & 0.000 \\
\hline Bosque El Olivar & 169.96 & 3.77 & 72 & 0.000 \\
\hline De Las Leyendas & 56.35 & -29.53 & 49 & 0.000 \\
\hline Zool. de Huachipa & 78.43 & -11.12 & 62 & 0.000 \\
\hline
\end{tabular}


Tabla 4. Caracterización de los suelos de los GPLM.

\begin{tabular}{lrrrrr}
\hline Parque & $\begin{array}{c}\% \\
\text { arcilla }\end{array}$ & $\begin{array}{c}\mathrm{pH} \\
(1: 1)\end{array}$ & $\begin{array}{c}\text { MO } \\
\%\end{array}$ & $\begin{array}{c}\text { C.E. } \\
\text { (extracto } \\
\text { saturación) } \\
\mathrm{dS} / \mathrm{m}\end{array}$ & $\begin{array}{c}\mathrm{CIC} \\
\mathrm{cmol}_{\mathrm{c}} \mathrm{kg}^{-1}\end{array}$ \\
\hline Campo de Marte & 13 & 7.47 & 11.31 & 1.25 & 30.68 \\
Bosque El Olivar & 9 & 7.06 & 3.67 & 3.45 & 16.8 \\
Pentagonito & 10 & 8.19 & 2.76 & 2.37 & 11.2 \\
Kennedy & 11 & 7.49 & 2.67 & 1.01 & 15.52 \\
Las Leyendas & 7 & 7.5 & 3.68 & 5.13 & 16.32 \\
Zoológico de Huachipa & 9 & 7.96 & 3.56 & 4.62 & 10.88 \\
\hline
\end{tabular}

contaminados con plomo; esto puede haber ocurrido por su exposición durante décadas al combustible como ha sido reportado por Laidlaw et al.(2017). Arosquipa et. al. (2013) observaron que el contacto de los seres vivos con estos suelos constituye un riesgo a la salud ya que este elemento pesado no cumple una función biológica y no se metaboliza.

La textura de los seis parques fue franco arenoso con ligeras variaciones en el porcentaje de arcilla mientras que el contenido de materia orgánica estuvo entre 2 y $4 \%$ a excepción del suelo de Campo de Marte con $11.31 \%$ de materia orgánica; suelos con mayor contenido de arcilla generalmente tienen mayor capacidad de intercambio catiónico (CIC) y mayor adsorción de plomo, la fracción materia orgánica puede incrementar la capacidad de cambio (He et al., 2017). Los suelos del Campo de Marte tienen el mayor contenido de arcillas, materia orgánica y CIC por lo que su adsorción de plomo debe ser mayor a pesar de que su clase textural sea semejante a los suelos de los otros parques. La actividad del plomo tiene relación directa con el $\mathrm{pH}$ de los suelos ya que al disminuir el $\mathrm{pH}$ disminuye la interacción del plomo con el suelo y aumenta la solubilidad influyendo en los procesos de adsorción y desorción (Amari et. al., 2017); los suelos de los parques Pentagonito y Zoológico de Huachipa tuvieron los mayores valores de $\mathrm{pH}$ por lo que se podría asumir existe una menor solubilidad del plomo a diferencia de los suelos del parque Bosque el Olivar que tuvo el menor valor de $\mathrm{pH}$ (Tabla 4).

La conductividad eléctrica se realizó en extracto de saturación y directo al suelo luego de 30 minutos de riego. Los valores de $\mathrm{CE}$ medidos directo al suelo mostraron incremento entre 0.3 y 0.6 unidades. Los resultados indican que los suelos de los parques Las Leyendas y Zoológico de Huachipa son ligeramente salinos mientras que Bosque El Olivar y Pentagonito son muy ligeramente salinos. Los valores de $\mathrm{CE}$ normalmente tienen una correlación directa con el contenido de plomo en los suelos (Solano, 2005).

\section{Conclusiones.}

Los suelos de los parques Campo de Marte, Bosque El Olivar y Pentagonito se encuentran contaminados con plomo siendo la media de plomo total de 225.59, 169.96 y 159.15 ppm respectivamente.
Los suelos del parque Zoológico de Huachipa, en la zona de "Bosque de Aves" (0.7 ha), tienen contaminación promedio por plomo de $266.41 \mathrm{ppm}$; el resto del parque (10.3 ha) no está contaminado (78.43 ppm).

Los suelos del parque Las Leyendas, en la zona "Pampa Galera" (8 ha), están contaminados por plomo en un nivel medio de $234.47 \mathrm{ppm}$; el resto del parque (90 ha) no está contaminado (56.35 ppm).

El parque Kennedy no tiene problema de contaminación de plomo en sus suelos siendo su media de $55.52 \mathrm{ppm}$.

\section{Agradecimientos.}

A los docentes del departamento académico de suelos de la Universidad Nacional Agraria La Molina (UNALM) por apoyar a que el laboratorio de análisis de suelos, plantas y fertilizantes (LASPAF-UNALM) financie los trabajos de investigación; a los trabajadores de los parques por su apoyo en la toma de datos en campo y a los representantes de las municipalidades y parques de los distritos de San Isidro, San Borja, Jesús María, Ate-Vitarte, San Miguel y Miraflores por el permiso concedido para el ingreso a los parques estudiados.

\section{Literatura citada.}

Amari T., Ghnaya T. \& Abdelly C. 2017. Nickel, cadmium and lead phytotoxicity and potential of halophytic plants in heavy metal extraction. South African Journal of Botany. July 2017, vol. 111, 99-110. Doi 10.1016/sajb.2017.03.011.

Arosquipa G. \& Villegas E. 2013. Determinación del contenido de plomo en sangre proveniente de la policía de tránsito femenina de las unidades Centro y Sur de Lima Metropolitana. Revista de la Sociedad Química del Perú. Volumen $79 \mathrm{n}^{\circ}$ 3: 229-235.

Autoridad Nacional del Agua. 2012. Estrategias para la protección de la calidad de los recursos hídricos de la Cuenca del Río Rímac.

Castillo Y. 2010. Evaluación toxicológica de plomo en suelos de Lima Metropolitana. UNMSM. Facultad de Farmacia y Bioquímica. Departamento Académico de Farmacología, Bromatología y Toxicología. Tesis para optar el título profesional de Químico Farmaceútico. 78h.

Doyle R. 1998. Effectiveness of soil amendments in reducing the bioaccessibility of lead, $\mathrm{Pb}$, on vacant lots in South Providence. Brown University.

El Comercio. 2015. Zoológico de Huachipa: uno de los 11 mejores de América Latina. 26 de octubre del 2015 
(https://elcomercio.pe/lima/zoologico-huachipa-11mejores-america-latina-235757). Perú.

He S., Lu Q., Li W., Ren Z., Zhou Z., Feng X., Zhang Y \& Li Y. 2017. Factors controlling cadmiun and lead activities in different parent material-derived soils from the Pearl River Basin. Chemosphere vol. 182: 509-516

Jackson M. 1964. Análisis químico de suelos. Barcelona (España): Ediciones Omega.

Ji P., Song Y., Leigh M. \& Liu Y. 2011. Strategies for enhancing the phytoremediation of cadmiumcontaminated agricultural soils by Solanum nigrum L. En Environmental Pollution 159:762-768.

Laidlaw M.A, Filippelli G.M., Brown S., Paz-Ferreiro J., Reichman S.M., Netherway P., Truskewycz A., Ball A.S. \& Mielke H.W. 2017. Case studies and evidence-based approaches to adressing urban soil lead contamination.

Martínez A G. \& Villafañe F. 1999. Intoxicación Aguda con Plomo en Bovinos. Revista ACOVEZ, Vol. 24 n.1, marzo 1999.

MINAM (Ministerio del Ambiente). 2014. Guía para Muestreo de Suelos. Perú. 38 p. (http://www.minam.gob.pe/wpcontent/uploads/2014/04/GUIA-MUESTREOSUELO_MINAM1.pdf).

MINAM (Ministerio del Ambiente). 2017. Estándares de Calidad Ambiental (ECA) para Suelo. DECRETO SUPREMO No 011-2017-MINAM. El Peruano; Lima, sábado 02 de diciembre del 2017; Año XXXIV - N $^{\circ}$ 14307:

$12-15$

(http://www.minam.gob.pe/consultaspublicas/wpcontent /uploads/sites/52/2017/07/Proyecto-de-DS_-ECASUELO.pdf). Perú.

Moronta-Riera J. \& Riverón-Zaldivar A.B. 2016. Evaluación de la calidad físico-química de las aguas y sedimentos de la costa oriental del lago de Maracaibo. En Minería y Geología del Instituto Superior Minero Metalúrgico de Moa. V32n2: 102-111.

Narváez J. 2014. Sistema de Irrigación y señoríos indígenas en el valle bajo del Rímac durante el siglo XVI. Boletín del Instituto Riva-Agüero (BIRA) No 37: 33-74.

OSINERGMIN (Organismo Supervisor de la Inversión en Energía y Minería). 2012. Resultados de los análisis realizados a los Gasoholes en las plantas de ventas.
OSINERGMIN (Organismo Supervisor de la Inversión en Energía y Minería). 1998. Aprueban el Reglamento de Infracciones y Sanciones al D.S.No.019-98-MTC que dispuso eliminar del mercado la oferta de Gasolina 95RON con plomo. RESOLUCIÓN 405-98-OS/CD. El Peruano; Lima, miércoles 4 de noviembre de 1998; AÑO XVI - Nº 6706: 165412-165413. Perú.

Pottier M., De La Torre V.S., Victor C., David L.C., Chalot M. \& Thomine S. 2015. Genotypic variations in the dynamics of metal concentrations in poplar leaves: A field study with a perspective on phytoremediation. En Environment Pollution 199: 73-82.

Sanín L.H., González-Cossio T., Romieu I. \& HernándezAvila M. 1998. Acumulación de plomo en huesos y sus efectos en la salud. Salud Pública Mex 40: 359-368.

Solano A. 2005. Movilización de metales pesados en residuos y suelos industriales afectados por la hidrometalurgia del cinc. Tesis Doctoral, Doctorado en Química, Facultad de Química, Departamento de Química Agrícola, Geología y Edafología. Universidad de Murcia, España. 370h.

Tepanosyan G, Maghakyan G, Sahakuan L. \& Saghatelvan A. 2017. Heavy metals pollution levels and children health risk assessment of Yerevan kindergartens soils. Ecotoxicology and environmental safety. Volume 142, august 2017. Pages 257-265.

Walkley A \& Black I. 1934. An examination of Degtjareff method for determining soil organic matter and a proposed modification of the chromic acid titration method. Soil Sci. 37:29-38.

Witzling L., Wander M. \& Phillips E. 2011. Testing and educating on urban soil lead: A case of Chicago community gardens. Journal of agricultura, food system, and community development.

Xu F., Liu Z, Cao Y., Qiu L., Feng J., Xu F. \& Tian X. 2017. Assessment of heavy metal contamination in urban river sediments in the Jiaozhou Bay catchment, Qingdao, China. En Elsevier Catena. Volume 150: 9-16.

Yang-Guang G. \& Yan-Peng G. 2016. Contamination, bioaccessibility and human health risk of heavy metals in exposed-lawn soils from 28 urban parks in southern Chipa's largest city, Guangzhou. Geochemestry.Volume 67, april 2016. Pages 52-58.

Anexo 1. Puntos de muestreo de identificación y detalle de los GPLM.

\begin{tabular}{llllllrr}
\multicolumn{2}{l}{ Campo de Marte: Coordenadas: Zona 18 L } & \multicolumn{4}{l}{ Coordenadas: Zona 18 L } \\
\multicolumn{2}{l}{ Muestreo de Identificación } & \multicolumn{1}{l}{$\mathrm{Pb}(\mathrm{ppm})$} & & \multicolumn{2}{l}{ Muestreo de Detalle } & $\mathrm{Pb}(\mathrm{ppm})$ \\
\hline CM1 & 278021 & 8665367 & 369.68 & $\mathrm{CM} 43$ & 277534 & 8664855 & 302.73 \\
CM2 & 277974 & 8665376 & 302.15 & $\mathrm{CM} 44$ & 277525 & 8664873 & 217.72 \\
CM3 & 277985 & 8665360 & 112.49 & $\mathrm{CM} 45$ & 277559 & 8664898 & 315.5 \\
CM4 & 278020 & 8665334 & 485.64 & CM46 & 277534 & 8664952 & 167.32 \\
CM5 & 277972 & 8665309 & 375.18 & CM47 & 277473 & 8664991 & 202.1 \\
CM6 & 278008 & 8665303 & 124.71 & CM48 & 277495 & 8665031 & 187.61 \\
CM7 & 277962 & 8665319 & 202.86 & CM49 & 277529 & 8665057 & 143.27 \\
CM8 & 277972 & 8665246 & 225.38 & CM50 & 277611 & 8665033 & 157.21 \\
CM9 & 277627 & 8665081 & 153.62 & CM51 & 278018 & 8665399 & 324.24 \\
CM10 & 277641 & 8665030 & 134.51 & CM52 & 277961 & 8665321 & 267.26 \\
CM11 & 277985 & 8665281 & 361.97 & CM53 & 277997 & 8665233 & 383.98 \\
\hline
\end{tabular}


Anexo 1. Puntos de muestreo de identificación y detalle de los GPLM.

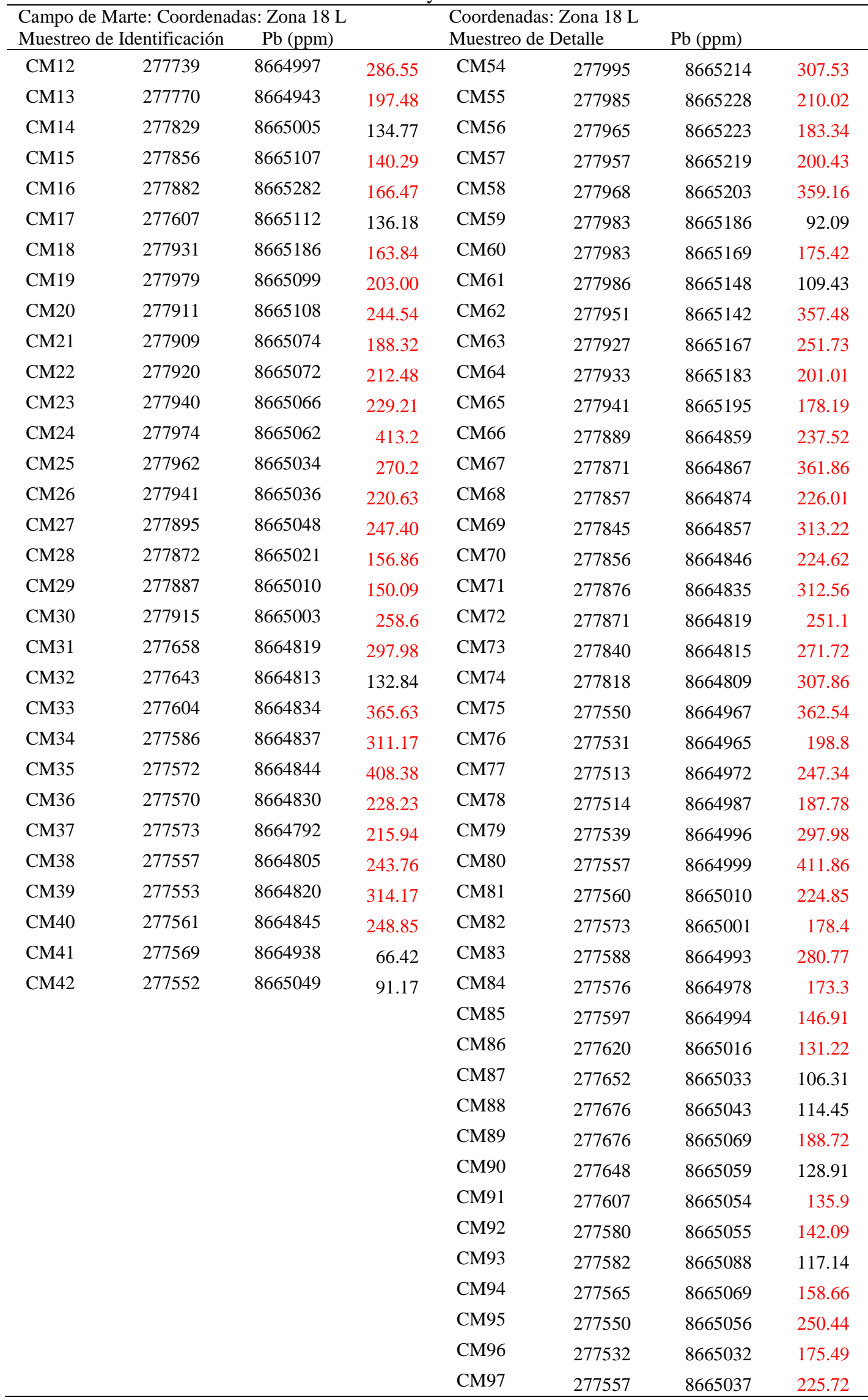


Anexo 1. Puntos de muestreo de identificación y detalle de los GPLM.

\begin{tabular}{|c|c|c|c|c|}
\hline \multirow[t]{2}{*}{$\begin{array}{l}\text { Campo de Marte: Coordenadas: Zona } 18 \mathrm{~L} \\
\text { Muestreo de Identificación } \quad \mathrm{Pb}(\mathrm{ppm})\end{array}$} & \multicolumn{2}{|c|}{$\begin{array}{l}\text { Coordenadas: Zona } 18 \mathrm{~L} \\
\text { Muestreo de Detalle }\end{array}$} & $\mathrm{Pb}(\mathrm{ppm})$ & \multirow[b]{2}{*}{98.05} \\
\hline & CM98 & 277554 & 8665011 & \\
\hline & CM99 & 277530 & 8665025 & 239.62 \\
\hline & CM100 & 277518 & 8665034 & 240.09 \\
\hline & CM101 & 277504 & 8665011 & 167.08 \\
\hline & CM102 & 277489 & 8665011 & 265.12 \\
\hline & CM103 & 277479 & 8664983 & 154.78 \\
\hline & CM104 & 277487 & 8664961 & 178.08 \\
\hline & CM105 & 277496 & 8664949 & 169.34 \\
\hline & CM106 & 277516 & 8664945 & 101.34 \\
\hline & CM107 & 277532 & 8664928 & 344.74 \\
\hline
\end{tabular}

Anexo 1. Puntos de muestreo de identificación y detalle de los GPLM.

\begin{tabular}{|c|c|c|c|c|c|c|c|}
\hline \multicolumn{2}{|c|}{ Bosque El Olivar } & \multirow{2}{*}{\multicolumn{2}{|c|}{$\begin{array}{l}\text { Coordenadas: Zona } 18 \mathrm{~L} \\
\text { ción } \quad \mathrm{Pb}(\mathrm{ppm})\end{array}$}} & \multicolumn{4}{|c|}{ Coordenadas: Zona 18 L } \\
\hline \multicolumn{2}{|c|}{ Muestreo de Identific } & & & Muestre & Detalle & $\mathrm{Pb}(\mathrm{ppm})$ & \\
\hline BO1 & 278509 & 8661792 & 127.76 & BO39 & 278487 & 8661346 & 181.47 \\
\hline $\mathrm{BO} 2$ & 278501 & 8661761 & 205.86 & BO40 & 278497 & 8661437 & 160.21 \\
\hline BO3 & 278484 & 8661708 & 176.78 & BO41 & 278510 & 8661536 & 327.74 \\
\hline BO4 & 278471 & 8661659 & 169.41 & BO42 & 278504 & 8661494 & 250.64 \\
\hline BO5 & 278461 & 8661599 & 256.24 & B043 & 278529 & 8661678 & 160.48 \\
\hline BO6 & 278466 & 8661640 & 154.79 & BO44 & 278529 & 8661703 & 191.99 \\
\hline $\mathrm{BO} 7$ & 278476 & 8661688 & 145.50 & BO45 & 278553 & 8661793 & 208.82 \\
\hline BO8 & 278495 & 8661778 & 197.89 & BO46 & 278613 & 8661834 & 169.89 \\
\hline BO9 & 278501 & 8661782 & 136.72 & BO47 & 278596 & 8661785 & 151.37 \\
\hline BO10 & 278430 & 8661440 & 111.39 & BO48 & 278563 & 8661641 & 300.54 \\
\hline BO11 & 278421 & 8661375 & 71.28 & BO49 & 278566 & 8661529 & 181.05 \\
\hline BO12 & 278445 & 8661522 & 74.13 & BO50 & 278558 & 8661475 & 133.31 \\
\hline BO13 & 278472 & 8661652 & 102.20 & BO51 & 278556 & 8661452 & 75.28 \\
\hline BO14 & 278414 & 8661322 & 270.75 & BO52 & 278548 & 8661376 & 110.07 \\
\hline BO15 & 278408 & 8661274 & 167.19 & BO53 & 278540 & 8661220 & 111.00 \\
\hline B016 & 278402 & 8661216 & 210.23 & BO54 & 278584 & 8661042 & 154.31 \\
\hline BO17 & 278390 & 8661102 & 124.13 & BO55 & 278603 & 8661063 & 186.53 \\
\hline B018 & 278450 & 8661414 & 149.58 & BO56 & 278637 & 8661209 & 155.59 \\
\hline BO19 & 278461 & 8661587 & 134.19 & BO57 & 278656 & 8661464 & 186.96 \\
\hline BO20 & 278386 & 8661062 & 229.56 & BO58 & 278688 & 8661606 & 147.30 \\
\hline BO21 & 278379 & 8660982 & 107.90 & BO59 & 278710 & 8661668 & 398.48 \\
\hline $\mathrm{BO} 22$ & 278457 & 8661057 & 123.55 & BO60 & 278698 & 8661637 & 329.04 \\
\hline $\mathrm{BO} 23$ & 278466 & 8661134 & 128.11 & BO61 & 278713 & 8661728 & 76.50 \\
\hline BO24 & 278474 & 8661209 & 136.52 & BO62 & 278705 & 8661801 & 281.15 \\
\hline $\mathrm{BO} 25$ & 278382 & 8660992 & 198.18 & BO63 & 278705 & 8661785 & 99.19 \\
\hline BO26 & 278480 & 8661250 & 209.93 & BO64 & 278699 & 8661810 & 172.97 \\
\hline BO27 & 278485 & 8661320 & 152.58 & BO65 & 278595 & 8661056 & 243.31 \\
\hline BO28 & 278481 & 8661291 & 96.00 & BO66 & 278619 & 8661097 & 165.99 \\
\hline BO29 & 278479 & 8661269 & 288.38 & BO67 & 278625 & 8661135 & 256.72 \\
\hline BO30 & 278476 & 8661229 & 212.37 & BO68 & 278630 & 8661170 & 270.21 \\
\hline BO31 & 278489 & 8661369 & 120.02 & BO69 & 278637 & 8661314 & 188.34 \\
\hline
\end{tabular}


Anexo 1. Puntos de muestreo de identificación y detalle de los GPLM.

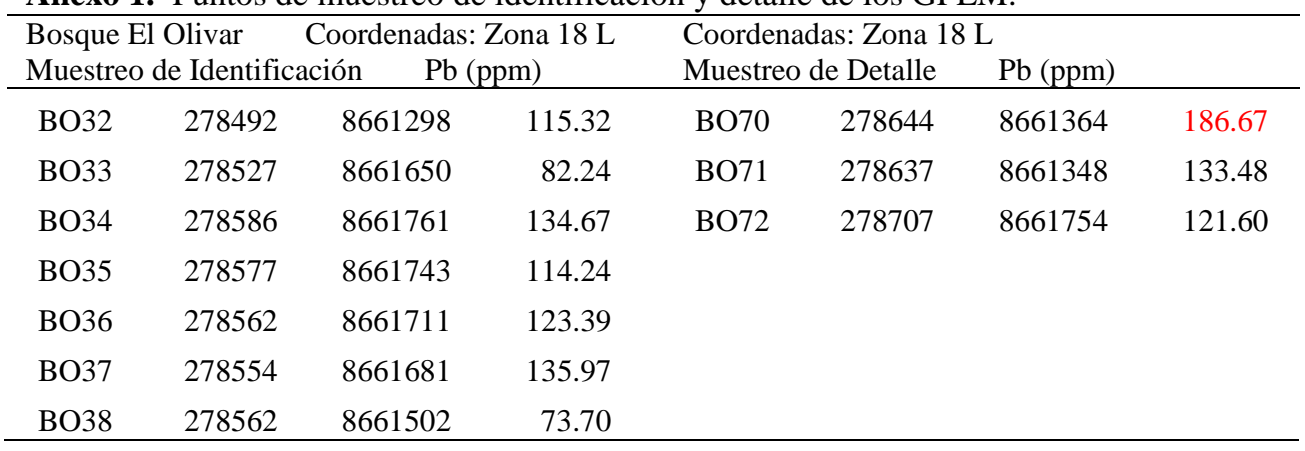

Anexo 1. Puntos de muestreo de identificación y detalle de los GPLM.

\begin{tabular}{|c|c|c|c|c|c|c|c|}
\hline Parqu & tagonito & Coordenad & na $18 \mathrm{~L}$ & Coorde & as: Zona & L & \\
\hline Mues & e Identificac & & & Muestr & le Detalle & $\mathrm{Pb}(\mathrm{ppm}$ & \\
\hline PP1 & 283922 & 8661932 & 190.37 & PP7 & 284023 & 8661894 & 140.07 \\
\hline PP2 & 284403 & 8661656 & 187.08 & PP8 & 284422 & 8661522 & 157.04 \\
\hline PP3 & 283554 & 8661560 & 189.64 & PP9 & 283960 & 8660924 & 186.76 \\
\hline PP4 & 283727 & 8660751 & 196.52 & PP10 & 283571 & 8660899 & 158.04 \\
\hline PP5 & 284402 & 8661271 & 154.21 & PP11 & 283534 & 8661436 & 187.53 \\
\hline PP6 & 283560 & 8661059 & 125.85 & PP12 & 283681 & 8661961 & 191.47 \\
\hline
\end{tabular}

Anexo 1. Puntos de muestreo de identificación y detalle de los GPLM.

\begin{tabular}{|c|c|c|c|c|c|c|c|}
\hline \multicolumn{2}{|c|}{ Parque Kennedy } & \multirow{2}{*}{\multicolumn{2}{|c|}{$\begin{array}{l}\text { Coordenadas: Zona } 18 \mathrm{~L} \\
\text { cción } \quad \mathrm{Pb}(\mathrm{ppm})\end{array}$}} & \multicolumn{3}{|c|}{ Coordenadas: Zona $18 \mathrm{~L}$} & \multirow[b]{3}{*}{37.36} \\
\hline \multicolumn{2}{|c|}{ Muestreo de Identificac } & & & Muestre & e Detalle & $\mathrm{Pb}(\mathrm{ppm})$ & \\
\hline PK1 & 279154 & 8659372 & 65.98 & PK20 & 279163 & 8659366 & \\
\hline PK2 & 278998 & 8659146 & 50.63 & PK21 & 279169 & 8659338 & 30.06 \\
\hline PK3 & 279157 & 8659368 & 71.89 & PK22 & 279165 & 8659320 & 74.33 \\
\hline PK4 & 278967 & 8659100 & 35.22 & PK23 & 279163 & 8659270 & 41.69 \\
\hline PK5 & 279087 & 8659258 & 70.58 & PK24 & 279158 & 8659262 & 66.95 \\
\hline PK6 & 279162 & 8659298 & 76.9 & PK25 & 279161 & 8659226 & 38.59 \\
\hline PK7 & 279144 & 8659314 & 30.79 & PK26 & 279149 & 8659207 & 38.26 \\
\hline PK8 & 279151 & 8659362 & 64.3 & PK27 & 279119 & 8659221 & 75.05 \\
\hline PK9 & 279105 & 8659291 & 54.8 & PK28 & 279036 & 8659211 & 7185 \\
\hline PK10 & 279137 & 8659230 & 31.45 & & 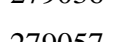 & 0650100 & 11.85 \\
\hline PK11 & 279132 & 8659241 & 52.3 & PK29 & 279057 & 8659188 & 65.54 \\
\hline PK12 & 279149 & 8659353 & 55.68 & PK30 & 279047 & 8659171 & 91.5 \\
\hline PK13 & 279136 & 8659359 & 148.49 & PK31 & 279027 & 8659136 & 73.59 \\
\hline PK14 & 279155 & 8659344 & 40.25 & PK32 & 278999 & 8659108 & 91.09 \\
\hline PK15 & 279154 & 8659334 & 40.98 & & & & \\
\hline PK16 & 279143 & 8659337 & 31.81 & & & & \\
\hline PK17 & 279127 & 8659340 & 71.18 & & & & \\
\hline PK18 & 279131 & 8659318 & 56.47 & & & & \\
\hline PK19 & 279127 & 8659288 & 48.93 & & & & \\
\hline
\end{tabular}

Anexo 1. Puntos de muestreo de identificación y detalle de los GPLM.

\begin{tabular}{lccrcrrr}
\hline \multicolumn{2}{c}{ Parque Las Leyendas } & \multicolumn{2}{c}{ Coordenadas: Zona 18 L } & \multicolumn{5}{c}{ Coordenadas: Zona 18 L } \\
\multicolumn{2}{l}{ Muestreo de Identificación } & Pb (ppm) & \multicolumn{5}{c}{ Muestreo de Detalle } \\
\hline PLL1 & 272798 & 8664994 & 78.4 & PLL51 & 272963 & 8664440 & 297.04 \\
PLL2 & 272811 & 8665039 & 63.15 & PLL52 & 272961 & 8664500 & 334.96 \\
PLL3 & 272817 & 8665100 & 71.07 & PLL53 & 272786 & 8665008 & 47.41 \\
\hline
\end{tabular}


Anexo 1. Puntos de muestreo de identificación y detalle de los GPLM.

\begin{tabular}{|c|c|c|c|c|c|c|c|}
\hline \multicolumn{4}{|c|}{$\begin{array}{l}\text { Parque Las Leyendas Coordenadas: Zona } 18 \mathrm{~L} \\
\text { Muestreo de Identificación } \quad \mathrm{Pb}(\mathrm{ppm})\end{array}$} & \multicolumn{4}{|c|}{$\begin{array}{l}\text { Coordenadas: Zona } 18 \mathrm{~L} \\
\text { Muestreo de Detalle } \quad \mathrm{Pb}(\mathrm{ppm})\end{array}$} \\
\hline PLL4 & 272834 & 8665134 & 92.87 & PLL54 & 273192 & 8664845 & 223.04 \\
\hline PLL5 & 272838 & 8665189 & 74.7 & PLL55 & 273202 & 8664837 & 334.98 \\
\hline PLL6 & 272850 & 8665234 & 33.83 & PLL56 & 273114 & 8664857 & 88.69 \\
\hline PLL7 & 272767 & 8665330 & 25.06 & & & & \\
\hline PLL8 & 272764 & 8665262 & 38.23 & & & & \\
\hline PLL9 & 272748 & 8665197 & 78.72 & & & & \\
\hline PLL10 & 272742 & 8665148 & 27.77 & & & & \\
\hline PLL11 & 272736 & 8665107 & 35.21 & & & & \\
\hline PLL12 & 272751 & 8665081 & 49.27 & & & & \\
\hline PLL13 & 272742 & 8665036 & 40.87 & & & & \\
\hline PLL14 & 272770 & 8664980 & 58.08 & & & & \\
\hline PLL15 & 272745 & 8665000 & 58.73 & & & & \\
\hline PLL16 & 272726 & 8665015 & 29.97 & & & & \\
\hline PLL17 & 272669 & 8664930 & 60.65 & & & & \\
\hline PLL18 & 272648 & 8664963 & 50.88 & & & & \\
\hline PLL19 & 272639 & 8664946 & 80.51 & & & & \\
\hline PLL20 & 272677 & 8664958 & 50.33 & & & & \\
\hline PLL21 & 273099 & 8665300 & 40.01 & & & & \\
\hline PLL22 & 273148 & 8665318 & 36.18 & & & & \\
\hline PLL23 & 273156 & 8665363 & 36.25 & & & & \\
\hline PLL24 & 273124 & 8665375 & 41.55 & & & & \\
\hline PLL25 & 273111 & 8665343 & 56.06 & & & & \\
\hline PLL26 & 273229 & 8664988 & 60.02 & & & & \\
\hline PLL27 & 273252 & 8664992 & 24.26 & & & & \\
\hline PLL28 & 273234 & 8665020 & 37.97 & & & & \\
\hline PLL29 & 273250 & 8665047 & 29.59 & & & & \\
\hline PLL30 & 273287 & 8665058 & 28.31 & & & & \\
\hline PLL31 & 273279 & 8664844 & 72.79 & & & & \\
\hline PLL32 & 273000 & 8664529 & 158.75 & & & & \\
\hline PLL33 & 272972 & 8664522 & 204.5 & & & & \\
\hline PLL34 & 273196 & 8664859 & 123.7 & & & & \\
\hline PLL35 & 273198 & 8664815 & 163.2 & & & & \\
\hline PLL36 & 273227 & 8664806 & 81.37 & & & & \\
\hline PLL37 & 273253 & 8664776 & 38.25 & & & & \\
\hline PLL38 & 273204 & 8664746 & 93.54 & & & & \\
\hline PLL39 & 273181 & 8664730 & 66.55 & & & & \\
\hline PLL40 & 273146 & 8664692 & 60.82 & & & & \\
\hline PLL41 & 273122 & 8664671 & 51.21 & & & & \\
\hline PLL42 & 273166 & 8664656 & 68.15 & & & & \\
\hline PLL43 & 273039 & 8664823 & 81.07 & & & & \\
\hline PLL44 & 273021 & 8664842 & 60.37 & & & & \\
\hline PLL45 & 273002 & 8664854 & 71.25 & & & & \\
\hline PLL46 & 272923 & 8664752 & 60.84 & & & & \\
\hline PLL47 & 272885 & 8664714 & 88.27 & & & & \\
\hline
\end{tabular}


Anexo 1. Puntos de muestreo de identificación y detalle de los GPLM.

\begin{tabular}{|c|c|c|c|c|c|}
\hline \multicolumn{4}{|c|}{ Parque Las Leyendas Coordenadas: Zona $18 \mathrm{~L}$} & \multicolumn{2}{|c|}{ Coordenadas: Zona $18 \mathrm{~L}$} \\
\hline \multicolumn{2}{|c|}{ Muestreo de Identificación } & \multicolumn{2}{|c|}{$\mathrm{Pb}(\mathrm{ppm})$} & Muestreo de Detalle & $\mathrm{Pb}(\mathrm{ppm})$ \\
\hline PLL48 & 273113 & 8664857 & 34.18 & & \\
\hline PLL49 & 273095 & 8664703 & 74.48 & & \\
\hline PLL50 & 272748 & 8664862 & 73.28 & & \\
\hline
\end{tabular}

Anexo 1. Puntos de muestreo de identificación y detalle de los GPLM.

\begin{tabular}{|c|c|c|c|c|c|c|c|}
\hline \multicolumn{4}{|c|}{$\begin{array}{l}\text { Parque Zoológico de Huachipa Coord: Zona } 18 \mathrm{~L} \\
\text { Muestreo de Identificación } \quad \mathrm{Pb}(\mathrm{ppm})\end{array}$} & \multicolumn{4}{|c|}{$\begin{array}{l}\text { Coordenadas: Zona } 18 \mathrm{~L} \\
\text { Muestreo de Detalle } \quad \mathrm{Pb}(\mathrm{ppm})\end{array}$} \\
\hline PZH1 & 293197 & 8671044 & 85.19 & PZH34 & 293321 & 8671158 & 110.51 \\
\hline $\mathrm{PZH} 2$ & 293246 & 8671059 & 284.85 & PZH35 & 293319 & 8671169 & 57.21 \\
\hline PZH3 & 293296 & 8671078 & 145.27 & PZH36 & 293330 & 8671165 & 36.92 \\
\hline PZH4 & 293310 & 8671085 & 39.79 & PZH37 & 293331 & 8671162 & 58.81 \\
\hline PZH5 & 293311 & 8671098 & 51.32 & PZH38 & 293345 & 8671165 & 67.60 \\
\hline PZH6 & 293297 & 8671094 & 31.91 & PZH39 & 293341 & 8671170 & 115.83 \\
\hline PZH7 & 293278 & 8671088 & 36.79 & PZH40 & 293350 & 8671175 & 91.12 \\
\hline PZH8 & 293278 & 8671063 & 157.11 & PZH41 & 293351 & 8671169 & 115.42 \\
\hline PZH9 & 293771 & 8671239 & 469.93 & PZH42 & 293362 & 8671175 & 79.58 \\
\hline PZH10 & 293291 & 8671099 & 22.45 & PZH43 & 293369 & 8671177 & 109.29 \\
\hline PZH11 & 293289 & 8671106 & 139.81 & PZH44 & 293375 & 8671182 & 72.61 \\
\hline PZH12 & 293380 & 8671145 & 315.15 & PZH45 & 293382 & 8671184 & 92.85 \\
\hline PZH13 & 293401 & 8671102 & 226.04 & PZH46 & 293413 & 8671193 & 78.61 \\
\hline PZH14 & 293282 & 8671109 & 103.37 & PZH47 & 293430 & 8671193 & 71.21 \\
\hline PZH15 & 293279 & 8671114 & 92.95 & PZH48 & 293448 & 8671194 & 86.39 \\
\hline PZH16 & 293284 & 8671121 & 41.45 & PZH49 & 293663 & 8671186 & 100.01 \\
\hline PZH17 & 293291 & 8671119 & 20.47 & PZH50 & 293643 & 8671197 & 87.56 \\
\hline PZH18 & 293305 & 8671133 & 136.75 & PZH51 & 293634 & 8671194 & 96.24 \\
\hline PZH19 & 293298 & 8671139 & 33.70 & PZH52 & 293637 & 8671183 & 78.00 \\
\hline PZH20 & 293472 & 8671175 & 265.63 & PZH53 & 293637 & 8671174 & 137.18 \\
\hline PZH21 & 293536 & 8671186 & 191.34 & PZH54 & 293733 & 8671215 & 330.91 \\
\hline PZH22 & 293660 & 8671180 & 248.5 & PZH55 & 293761 & 8671232 & 134.35 \\
\hline PZH23 & 293467 & 8671193 & 171.52 & PZH56 & 293291 & 8671143 & 27.94 \\
\hline PZH24 & 293469 & 8671188 & 384.97 & PZH57 & 293303 & 8671146 & 36.66 \\
\hline PZH25 & 293649 & 8671173 & 222.97 & PZH58 & 293306 & 8671139 & 32.26 \\
\hline PZH26 & 293319 & 8671136 & 74.31 & PZH59 & 293310 & 8671131 & 35.19 \\
\hline PZH27 & 293314 & 8671145 & 34.35 & PZH60 & 293515 & 8671104 & 46.08 \\
\hline PZH28 & 293313 & 8671151 & 62.56 & PZH61 & 293446 & 8671111 & 111.32 \\
\hline PZH29 & 293306 & 8671154 & 70.51 & PZH62 & 293407 & 8671139 & 108.83 \\
\hline PZH30 & 293309 & 8671154 & 60.03 & PZH63 & 293380 & 8671065 & 99.01 \\
\hline PZH31 & 293313 & 8671157 & 48.43 & PZH64 & 293434 & 8671051 & 98.96 \\
\hline PZH32 & 293438 & 8671153 & 203.16 & PZH65 & 293409 & 8671076 & 76.05 \\
\hline \multirow[t]{5}{*}{ PZH33 } & 293312 & 8671151 & 56.19 & PZH66 & 293373 & 8671087 & 44.78 \\
\hline & & & & PZH67 & 293363 & 8671092 & 43.75 \\
\hline & & & & PZH68 & 293358 & 8671097 & 45.96 \\
\hline & & & & PZH69 & 293350 & 8671114 & 49.85 \\
\hline & & & & PZH70 & 293320 & 8671070 & 63.92 \\
\hline
\end{tabular}


Anexo 1. Puntos de muestreo de identificación y detalle de los GPLM.

\begin{tabular}{llrrrr}
\hline Parque Zoológico de Huachipa Coord: Zona 18 L & \multicolumn{4}{l}{ Coordenadas: Zona 18 L } \\
Muestreo de Identificación & Pb (ppm) & Muestreo de Detalle & Pb (ppm) & \\
\hline & PZH71 & 293322 & 8671073 & 109.08 \\
& PZH72 & 293309 & 8671063 & 138.71 \\
& PZH73 & 293285 & 8671090 & 77.57 \\
& PZH74 & 293280 & 8671096 & 44.00 \\
& PZH75 & 293708 & 8671242 & 222.61 \\
& PZH76 & 293396 & 8671141 & 246.18 \\
& PZH77 & 293421 & 8671155 & 362.94 \\
\hline
\end{tabular}

${ }^{1}$ Doctorante de la Universidad Nacional Mayor de San Marcos y docente de la Universidad Nacional Agraria La Molina. Av. La Molina s/n, Lima 12, Perú. 1tello@lamolina.edu.pe

2 Docente de la Universidad Nacional Mayor de San Marcos y asesor de tesis.

${ }^{3}$ Docente de la Universidad Nacional Agraria La Molina y doctorante de la Universidad Nacional Mayor de San Marcos. 